\title{
Concepções, desafios e competências dos enfermeiros em cuidados paliativos na atenção primária à saúde
}

RESUMO | Objetivo: identificar conhecimento, competências e desafios enfrentados pelos enfermeiros das Estratégias de Saúde da Família acerca dos cuidados paliativos. Método: estudo exploratório qualitativo realizado no segundo semestre de 2018 com 24 enfermeiros atuantes em 24 municípios do Rio Grande do Sul. A coleta de dados foi realizada por meio de um questionário online e analisado pela análise temática. Resultados: sugerem que os principais desafios compreendem conhecimento incipiente sobre a temática, falta de preparo técnico e científico e a ausência de uma equipe multiprofissional nos serviços que atuam. Relacionado às competências necessárias, destacaram-se o planejamento e execução do cuidado, ter conhecimento técnico e científico e estabelecer um plano de cuidado integral ao paciente. Conclusão: Acredita-se que irá auxiliar gestores municipais de saúde a perceberem os cuidados paliativos como estratégias na viabilização, condução e implementação de propostas inovadoras de cuidados alicerçadas nos preceitos desta prática, possibilitando ações propositivas aos usuários.

Palavras-chaves: Cuidados paliativos; Atenção Primária à Saúde; Enfermagem em saúde comunitária.

ABSTRACT | Objective: to identify knowledge, skills and challenges faced by nurses in Family Health Strategies about palliative care. Method: qualitative exploratory study carried out in the second semester of 2018 with 24 nurses working in 24 municipalities in Rio Grande do Sul. Data collection was performed through an online questionnaire and analyzed by thematic analysis. Results: they suggest that the main challenges comprise incipient knowledge on the theme, lack of technical and scientific preparation and the absence of a multidisciplinary team in the services they operate. Regarding the necessary skills, care planning and execution, technical and scientific knowledge and establishing a comprehensive patient care plan stood out. Conclusion: It is believed that it will help municipal health managers to perceive palliative care as strategies in the feasibility, conduction and implementation of innovative care proposals based on the precepts of this practice, enabling propositional actions to users.

Keywords: Palliative care; Primary Health Care; Community health nursing.

RESUMEN | Objetivo: identificar conocimientos, habilidades y desafíos que enfrentan los enfermeros en Estrategias de Salud de la Familia sobre cuidados paliativos. Método: estudio cualitativo exploratorio realizado en el segundo semestre de 2018 con 24 enfermeras trabajando en 24 municipios de Rio Grande do Sul. La recolección de datos se realizó a través de un cuestionario en línea y se analizó mediante análisis temático. Resultados: sugieren que los principales desafíos comprenden el conocimiento incipiente sobre el tema, la falta de preparación técnica y científica y la ausencia de un equipo multidisciplinario en los servicios que operan. En cuanto a las competencias necesarias, se destacó la planificación y ejecución de la atención, contar con conocimientos técnicos y científicos y establecer un plan integral de atención al paciente. Conclusión: Se cree que ayudará a los gestores de salud municipales a percibir los cuidados paliativos como estrategias en la viabilidad, conducción e implementación de propuestas de atención innovadoras basadas en los preceptos de esta práctica, posibilitando acciones proposicionales a los usuarios.

Palabras claves: Cuidados paliativos; Primeros auxilios; Enfermería en salud.

\section{Camila Mumbach de Melo}

Enfermeira. Residente em Urgência e emergência/Intensivismo Hospital de Clínicas de Passo Fundo. Passo Fundo/RS.

ORCID: 0000-0003-4170-7128

\section{Kelly Meller Sangoi}

Enfermeira Oncológica, Intensivista e Paliativista. Mestre em Ciências da Saúde PUC/RS. Universidade Regional Integrada do Alto Uruguai e das Missões. Campus Santo Ângelo. Santo Ângelo/RS.

ORCID: 0000-0003-4909-0660

\section{Janaina Kunzler Kochhann}

Enfermeira. Pós-Graduada em saúde da Família UNINTER. Universidade Regional Integrada do Alto Uruguai e das Missões. Campus Santo Ângelo. Santo Ângelo/RS. ORCID: 0000-0003-2360-2170

\section{Lilian Zielke Hesler}

Enfermeira. Doutora em Enfermagem UFRGS. Universidade Regional Integrada do Alto Uruguai e das Missões. Campus Santo Ângelo. Santo Ângelo/RS. ORCID: 0000-0001-9363-2709

\section{Rosane Teresinha Fontana}

Enfermeira. Doutora em Enfermagem UFRGS. Universidade Regional Integrada do Alto Uruguai e das Missões. Campus Santo Ângelo. Santo Ângelo/RS.

ORCID: 0000-0001-6480-9624

Recebido em: 08/03/2021

Aprovado em: 28/04/2021
INTRODUÇÃO

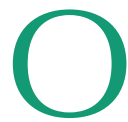
s Cuidados Paliativos (CP) são um direito inalienável dos cidadãos e focam no controle de questões funcionais e sintomáticas complexas que propõem mudanças no modo de cuidar do paciente com doença sem possibilidades terapêuticas para a cura. Esse modelo de atenção propõe romper com o foco tradicional, de ênfase na doença, para o cuidado integral a partir da ativa participação do paciente e familiares na tomada de decisão'.

Os $\mathrm{CP}$ envolvem práticas que buscam aumentar a qualidade de vida dos pacientes por meio da prevenção e alívio do 
sofrimento. Esse processo envolve a identificação precoce de suas necessidades, o tratamento da dor, dos problemas físicos, psicossociais e espirituais detectados a partir de um olhar sensível e acolhedor. Durante os CP é importante a utilização de uma abordagem que contemple o enfrentamento da morte como um processo natural, sobretudo com ações interprofissionais, conforme recomendações da Organização Mundial de Saúde (OMS)².

Os CP são mais efetivos quando iniciados precocemente, ou seja logo após o diagnóstico. Desta forma é possível minimizar o sofrimento, dar suporte e proporcionar um sistema de apoio capaz de ajudar o paciente a viver ativamente durante o tempo que lhe resta, além de amparar a família no processo da doença e luto ${ }^{3}$.

Os CP se apresentam em um crescimento expressivo no Brasil, visto que o marco principal ocorreu por meio da Resolução No 41, de 31 de outubro de 2018, que objetivou organizar os cuidados paliativos continuados no âmbito do Sistema Único de Saúde (SUS) ${ }^{4}$. Porém, ainda existem objeções que vão em contraposição ao desenvolvimento desses cuidados, devido às instituições de saúde sofrerem influências de um modelo de saúde voltado a prática curativista, em que o interesse principal é curar. Por ser uma temática ainda incipiente em nosso contexto social, os CP são negligenciados em muitas instituições, comprometendo a qualidade da assistência ao paciente ${ }^{3}$.

Os principais níveis de atenção dos $\mathrm{CP}$ categorizam-se em: cuidados no domicílio, atendimento ambulatorial, hospitalar ou procedimentos em leito-dia e internação hospitalar ${ }^{5}$. Dentre eles destaca-se o atendimento domiciliar como uma modalidade adotada no âmbito dos sistemas de saúde, contribuindo para o bem-estar, promoção do autocuidado, fornecendo suporte ao paciente e seus familiares frente às suas novas necessidades ${ }^{6}$.

A atenção domiciliar é uma modalidade de atenção à saúde complementar às já existentes, caracterizada por um conjunto de ações de assistência à saúde prestada em domicílio, garantida a pacientes que necessitam de CP e atribuída a partir da intensidade do cuidado com restrição ao leito ou a domicilio. Esta atenção tem por vantagens a realização da prevenção precoce de complicações, educação em saúde, conforto, contribuições para a rotina do paciente, e o vínculo familiar, conjuntamente a desospitalização, constituindo-se um dos eixos centrais da assistência domiciliar.
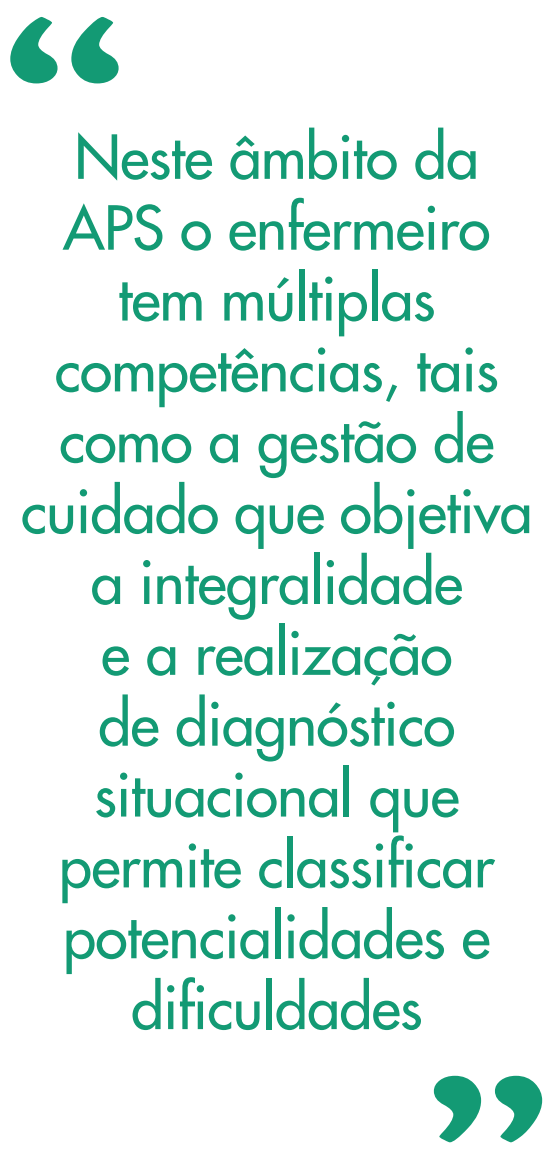

No cenário nacional esses cuidados abrangem a Atenção Primária a Saúde (APS), que é "porta de entrada" do sistema, ponto de atenção principal que concebe uma equipe multidisciplinar, construída de forma a reorganizar os serviços de saúde e garantir o acesso universal e integral à população ${ }^{7}$.

Visando a reorganização da APS foi criada a Estratégia Saúde da Família (ESF) como plano de extensão, consolidação e qualificação da APS, que aponta o entendimento do processo saúde/doença, propondo solucionar as necessidades individuais e coletivas de uma população. Há o direcionamento para a universalização dos cuidados primários agregando princípios fundamentais para uma APS abrangente, respeitando o princípio da equidade e da integralidade da atenção, com enfoque familiar, que valoriza o acoIhimento, o vínculo, a humanização e a orientação comunitária ${ }^{8}$.

Neste âmbito da APS o enfermeiro tem múltiplas competências, tais como a gestão de cuidado que objetiva a integralidade e a realização de diagnóstico situacional que permite classificar potencialidades e dificuldades ${ }^{9,10}$.

Diante de uma análise do crescente número de idosos e o aumento da prevalência de doenças crônicas a nível nacional e internacional, é notório que a omissão de ações implicadas como os CP se estabelecem como um problema de saúde pública em nosso país. Estima-se que no Brasil ocorra um milhão de óbitos anualmente, dos quais mais da metade, 650 mil são ocasionados por doenças crônicas ${ }^{11}$. Essa situação resulta em uma maior quantidade de pessoas que necessitam destes cuidados, no entanto ainda uma minoria dos pacientes se beneficiam com os $\mathrm{CP}$, principalmente em países em desenvolvimento ${ }^{12}$.

Perante essa problemática, há necessidade de uma inserção proativa dos CP no ambiente que o paciente frequenta, notadamente na APS, pois representam importantes estratégias na perspectiva de qualificar o viver e o morrer no mundo contemporâneo, a partir de um olhar amplo para os aspectos que envolvem a complexidade do ser humano. Diante desta contextualização emerge a seguinte questão de pesquisa: quais as concepções e dificuldades dos enfermeiros de estratégias de saúde da família sobre cuidados paliativos?

Esse estudo tem por objetivo identificar junto aos enfermeiros de unidades de Estratégia de Saúde da Família quais são as 
concepções, desafios e competências para a abordagem dos cuidados paliativos.

\section{MÉTODO}

Trata-se de um estudo exploratório, de natureza qualitativa ${ }^{13}$. A pesquisa ocorreu no segundo semestre de 2018 . Os participantes foram 24 enfermeiros que atuavam em ESF de municípios pertencentes a uma região de saúde compostas de 24 municípios do Estado do Rio Grande do Sul. Os critérios de inclusão foram: ser graduado em enfermagem; trabalhar em ESF dos municípios que compõem a região de saúde do RS.

A coleta de dados realizou-se por meio de um questionário semiestruturado com perguntas abertas e fechadas, utilizando a ferramenta virtual Google Docs (questionário online) desenvolvido pelos próprios autores. O Google Docs permite a formulação de questionários para realização de trabalhos científicos, diminuindo barreiras geográficas, facilitando o seu compartilhamento com público amplo.

Os enfermeiros foram convidados a participar da pesquisa de maneira informal, mediante contato telefônico em todas as ESF, divulgando a pesquisa, momento em que foi apresentado o projeto e seus objetivos e perguntando sobre interesse em participar do estudo. Assim, que o interesse era manifestado pelo participante era solicitado que fornecesse um endereço de e-mail para envio do TCLE e do questionário. Inicialmente foram convidados a participar 24 enfermeiros (um de cada município), contudo, dois se recusaram a participar, havendo necessidade de entrar em contato com outros dois profissionais de dois municípios diferentes. Responderam ao questionário (um de cada cidade da região de saúde). Após as respostas houve a devolutiva dos questionários, os quais compuseram o corpus da análise.

O estudo respeitou a Resolução 466/12 do Conselho Nacional de Saúde, que regulamenta a pesquisa em seres humanos. O projeto foi desenvolvido me- diante parecer favorável 2.899.192 do Comitê de Ética e Pesquisa da Universidade Regional Integrada do Alto Uruguai e das Missões - Campus Santo Ângelo/RS e dos secretários municipais de saúde dos 24 municípios de uma Região de Saúde do RS, mediante a assinatura da Declaração de Instituição coparticipante. Diante do recebimento dos questionários, foi garantido anonimato ao participante, os quais serão identificados, nesse estudo, pela abreviação da palavra enfermeiro (ENF) e pela sequência numérica de 1 a 24 .

O processo de análise de dados, juntamente com a discussão e a interpretação destes, foi orientado pela técnica de análise de conteúdo das falas, na modalidade temática14. Da análise emergiram duas categorias temáticas: Cuidados Paliativos: concepções dos enfermeiros na ESF e Desafios, competências e contribuições do enfermeiro frente aos cuidados paliativos na ESF.

\section{RESULTADOS}

\section{Caracterização dos participantes}

Os participantes foram identificados quanto ao sexo, idade, tempo de atuação. Houve predominância do sexo feminino com $87.5 \%(n=21)$. A idade média foi de 36,1 anos e a média de tempo de atuação na área da enfermagem foi de 10,5 anos.

Cuidados Paliativos: concepções dos enfermeiros na ESF

Todos os participantes responderam que conheciam o conceito de $\mathrm{CP}$, entretanto, as definições apresentadas mostraram-se incompletas ou equivocadas. Apenas dois respondentes descreveram a definição correta dos $\mathrm{CP}$, que abrange as dimensões físicas, sociais, psicológicas e espirituais, a avaliação e tratamento para alivio da dor. Três entenderam os CP em dimensões fragmentadas com o foco assistencial e controle de sintomas, conforme definições a seguir:

(ENF 01) São cuidados destinados a pacientes para alívio de sintomas, com alguma doença incurável.

(ENF 11) Entendo por cuidados paliativos as práticas assistenciais usadas em pacientes com doenças terminais.

(ENF 17) Cuidados paliativos promove assistência ao paciente terminal, naquele momento sobre sua necessidade para estar no local de atendimento.

E 19 dos participantes não souberam ou optaram por não definir os $\mathrm{CP}$, demonstrando lacunas conceituas nos saberes, destes trabalhadores, sobre os cuidados paliativos.

Outro aspecto relevante a partir das respostas é a associação com a terminalidade. Existe uma tendência de os profissionais associarem os CP somente aos pacientes em fase terminal, que pode ser vista nas citações anteriores e conforme esta resposta:

(ENF 24) Entendo por cuidados paliativos as práticas de assistência usadas em pacientes com doenças terminais.

Alguns profissionais que desconhecem amplamente os CP pressupõem que no processo de terminalidade não se tenha mais o que fazer, conforme as falas:

(ENF-2) [...] Muitas vezes o profissional se depara com pacientes que já se encontram em fase terminal, impossibilitando o desenvolvimento de estratégias que possibilitariam maior qualidade de vida $[. .$.

(ENF- 15) Desafios é uma palavra que encontramos todos os dias. Mas o grande desafios encaramos desde quando recebemos o paciente, é a resposta ao tratamento diminuindo conforme os dias, e você vê que não consegue fazer mais nada, além de esperar.

Quanto as patologias aplicáveis aos 
$\mathrm{CP}$, seis demonstraram saber as doenças e sete participantes citaram que são cuidados destinados apenas a pacientes oncológicos.

(ENF- 1) Câncer, AVC.

(ENF- 3) Câncer.

(ENF-5) Pacientes oncológicos.

(ENF-6) Oncológicas,

(ENF-24) Pacientes acamados e oncológicos

Os demais trouxeram tanto doenças aplicáveis como as inaplicáveis, configurando um conhecimento ainda incipiente sobre as doenças que se enquadram nestes cuidados.

Desafios, competências e contribuições do enfermeiro frente aos cuidados paliativos na ESF

Os enfermeiros descreveram alguns desafios, competências e contribuições relacionadas aos $\mathrm{CP}$ em ESF, mas que também podem ser atribuídos de modo geral a todos os âmbitos da APS.

Inicialmente, os profissionais foram questionados a respeito de seu contato com pacientes em CP e se prestavam cuidados a esses pacientes. A grande maioria respondeu que já cuidaram ou cuidam destes pacientes, apontando sobre a grande demanda de pacientes que necessitam desses cuidados na APS.

No que corresponde aos desafios enfrentados pelos enfermeiros, foi indicado como fator principal o desconhecimento e a insegurança diante dos CP.

(ENF-1) Os desafios são em conhecer a doença e quais cuidados aplicar.

(ENF-6) Falta de conhecimento.

(ENF-7) Necessita ser discutido mais sobre este tema.

(ENF-9) A falta de qualificação em certas práticas e mesmo o entendimento dos cuidados paliativos dificultam o processo.

Curiosamente, este resultado foi ob- tido após a maioria dos participantes terem mencionado conhecimento sobre os CP. Nesse sentido grande parte dos enfermeiros responderam que receberam informações sobre CP durante a graduação. Referente a questão de preparo técnico e teórico para controles de sintomas comuns como dor, dispneia e fadiga, três quartos dos participantes responderam que tiveram esse preparo na graduação, situação que sinaliza avanço na formação acadêmica.

O processo de compreender a finitude da vida também é apontado como desafio pois é um fenômeno complexo para todos os envolvidos. Além disso, existe uma dificuldade em auxiliar no entendimento dos CP pelos familiares, como citado nas falas:

(ENF-8) O maior desafio é compreender o fim da vida [...].

(ENF-9) Fazer com que a família entenda e ajude no processo dos cuidados paliativos.

(ENF-10) Lidar com a morte e o morrer. Fragilidade da familiar e paciente.

(ENF-23) Os desafios maiores seria tanto a resistência do paciente em aceitar a doença, como os demais familiares.

Outro desafio está relacionado a ausência de uma equipe multidisciplinar completa; somente a equipe mínima de uma ESF muitas vezes não consegue trabalhar com todas as demandas que o paciente em CP requer.

(ENF-17) Os cuidados paliativos vão além da equipe mínima do ESF, precisamos do entendimento de todos os profissionais que fazem parte da Atenção Básica tais como: Gestor, Psicólogo, Nutricionista, Fisioterapeuta, Assistente Social, Farmacêutico [...]

(ENF-17) ter ajuda de uma equipe multidisciplinar nesses cuidados. (ENF-20) Necessitamos de apoio de mais profissionais da saúde não só da enfermagem, mas de outras áreas.

Também é apontado como dificuldade o início tardio dos cuidados paliativos, a falta de retorno dos outros níveis de atenção, pois esse nível de atenção não abrange os cuidados de alta especialidade, havendo a necessidade muitas vezes dos cuidados serem dimensionados a outro nível de atenção.

(ENF-9) Em minha prática na Atenção Básica penso que a falta de comunicação e contra referência desse paciente na Rede de atenção, dificultam bastante o atendimento, a coordenação do cuidado;

(ENF-13) Prontuários de referência e contra referência aos pacientes oncológicos.

Como competências do enfermeiro foi apontada a capacidade de promover o cuidado, ter conhecimento técnico e científico, estabelecer um plano de cuidado integral ao paciente analisando as necessidades sociais e espirituais, mas também as físicas, visando auxiliar no manejo da dor de forma ampla fornecendo orientações e ações que promovam a qualidade de vida. Algumas das competências apresentadas:

(ENF-2) O profissional de enfermagem tem um papel relevante na equipe de cuidados paliativos, considerando sua posição privilegiada de permanecer a maior parte do tempo junto à pessoa enferma e poder prestar a maior parcela de cuidados, além de poder posicionar-se como intermediador entre a pessoa/família e os demais membros da equipe.

(ENF-4) Dentre as competências o enfermeiro deve ter conhecimento para avaliar de forma sistemática a clínica do pacien- 
te, estabelecendo prioridades. Além da clínica deve conhecer as condições biopsicossociais do paciente e sua família. O conhecimento técnico para a realização dos procedimentos deve incorporar o olhar holístico e integral. A comunicação, seja ela verbal ou não verbal é fundamental para o estabelecimento de vínculo e a identificação de necessidades do paciente e de seus familiares[...]. (ENF-16) Na equipe multidisciplinar de cuidados paliativos, os profissionais de enfermagem estão na linha de frente para prover cuidado, conforto e aconselhamento de famílias e pacientes. Nesta interação, o sucesso na execução do cuidado advém da relação estabelecida entre paciente-enfermagem e do interesse e vontade desses para exercer os cuidados ao fim da vida.

É também descrito por um dos profissionais como competências do enfermeiros vinculados aos $\mathrm{CP}$ :

(ENF-19) Ser profissional especialista na área, ter perfil para cuidados paliativos.

(ENF-20) Ter Conhecimento sobre o assunto para desenvolver de forma correta.

Outra competência citada foi o cuidado na atenção domiciliar.

(ENF-5) [...] juntamente com a equipe multiprofissional planejar os cuidados que o paciente necessita melhorando sua qualidade de vida, envolvendo a família e utilizando atenção domiciliar como ferramenta.

Portanto, a partir dos desafios e competências vinculados aos $\mathrm{CP}$, observa-se que a enfermagem busca contribuir para a realização desses cuidados por meio de orientações e visitas domiciliares. Constatou-se que as contribuições descritas pelos participantes da pesquisa foram semelhantes as competências do profissional enfermeiro em ESF, conforme relatos:

(ENF-4) Programando as visitas domiciliares da equipe aos pacientes que necessitam de cuidados paliativos, orientar e ter habilidade para dialogar com os membros da equipe em relação à dor, ao sofrimento e à morte. Explicar que o papel da equipe de saúde é proporcionar conforto, segurança, atender as necessidades básicas dos pacientes, ampliando o olhar para o holístico e integral. Dialogar com empatia com o paciente e sua família para a formação do vínculo.

(ENF- 10) Conhecer primeiramente toda a sua população adscrito e seus problemas, para poder elaborar os planos de cuidados. Lembrando que toda a equipe é responsável pelos cuidados do paciente.

(ENF-22) [...] Pacientes quando em seus domicílios necessitam muito de orientação e apoio por parte do ESF.

(ENF-24) importante ensinar os cuidadores e família, para que possa assistir esse paciente contemplando todas as necessidades.

Por fim, quando os participantes foram questionados a respeito de seu interesse na atividade de educação permanente sobre CP vinculadas a ESF, a quase totalidade dos enfermeiros responderam que consideram tal atividade relevante para sua prática. Entende-se que a educação a esses profissionais é um fator de extrema relevância, que agregará grandes contribuições a esses cuidados e, portanto, são necessárias intervenções mais propositivas, dinâmicas e que gerem impacto nas necessidades de saúde do usuário, no âmbito da APS.

\section{DISCUSSÃO}

As atribuições relacionadas aos $\mathrm{CP}$ mostram a incipiência do significado referido a esses cuidados. Ainda que grande parte dos profissionais relataram saber e ter recebido informações durante a graduação foi possível perceber, por meio da conceitualização sobre a temática, que os profissionais apresentam conhecimento incompleto e/ou deficitário sobre CP. Um dos fatores que contribuem para essa situação são as lacunas no processo ensino e aprendizagem quanto à socialização de saberes sobre os $\mathrm{CP}$ nos componentes curriculares, durante a graduação, consequentemente, os profissionais ao saírem da formação acadêmica encontram-se despreparados frente a esta realidade ${ }^{15}$.

As associações fragmentadas, como a relação dos $\mathrm{CP}$ somente ao processo de terminalidade, reforçam as deficiências do conhecimento dos profissionais, sobretudo ao considerarmos que esses cuidados devem estar presentes durante todo o percurso da doença, não exclusivamente no processo do final de vida, pois quanto mais cedo iniciados, maior as chances de proporcionar qualidade de vida durante o percurso da doença ${ }^{16}$. Além disso, a associação de CP apenas a pacientes oncológicos também sugere a fragmentação do conhecimento, uma vez que os CP abrangem várias outras doenças ${ }^{17}$.

Se partirmos do pressuposto de que apenas profissionais da área, ou seja, especialistas deverão cuidar destes pacientes, a maioria da população que necessita de CP continuará desassistida. Consequentemente os profissionais envolvidos neste contexto devem estar aptos a estes cuidados. Mesmo não sendo especialista deve ter um amplo conhecimento sobre a temática, para aplicar os CP corretamente, seguindo os princípios do mesmo.

Portanto, observa-se a necessidade de educação permanente dessas equipes na APS, e a partir de amplas reflexões discussões conceituais, diagnósticas e terapêuticas sobre esse assunto, desenvolver estratégias de cuidado de acordo com 
os princípios dos $\mathrm{CP}^{17}$. Nesse sentido é fundamental instigar a formação de profissionais tanto no ensino de graduação, como no de pós-graduação, além da e educação permanente ${ }^{4}$.

Quanto as competências do enfermeiro, segundo a Portaria $n^{\circ} 2.436$ de 21 de setembro de 2017, da Política Nacional de Atenção Básica, destaca que dentre as atribuições do profissional enfermeiro na APS, estão a elaboração de um plano de cuidado para pessoas que possuem doenças crônicas, junto a equipe multidisciplinar, a escuta qualificada e classificação de risco, o planejar, gerenciar e avaliar as atividades ${ }^{8}$.

O profissional enfermeiro contribui significativamente com os $\mathrm{CP}$, todavia ele não desenvolve os cuidados sozinho, para isso conta uma equipe multidisciplinar. Entretanto a equipe mínima de ESF compõem-se no mínimo por um médico, um enfermeiro, um auxiliar e/ou técnico de enfermagem, agente comunitário de saúde (ACS), e pode ter um cirurgião-dentista e auxiliar ou técnico em saúde bucal $^{8}$, caracterizando um grande desafio, pois há necessidade de cuidados amplos, visto que esses se interligam e são de grande importância para assistência de qualidade prestada ao paciente.

Embora a ESF não tenha a equipe completa para $\mathrm{CP}$, é preciso garantir esses cuidados com as condições atuais e buscar outras estratégias para proporcionar a qualidade do cuidado, seja articulando com outros níveis de atenção, ou outras redes de cuidado ${ }^{18,19}$. É a partir de problemas, demandas e necessidades de saúde de pessoas e grupos sociais em seus territórios, assim como dificuldades profissionais e de todos os tipos de equipe que atuam na APS que se estabelece o processo de trabalho. Para isto são necessárias praticas intersetoriais, de gestão, compartilhamento de conhecimentos, de cuidado em rede e educação permanente em territórios que a equipe é responsável ${ }^{8}$.

O desenvolvimento da comunicação, também é posto como um desafio, visto que é um elemento de grande importân- cia para os $\mathrm{CP}$ de qualidade, pois permitir o esclarecimento de dúvidas contribuindo nos cuidados direcionando aos ideais, bem como a formação de vínculo com o paciente, familiares, engajando o cuida$\mathrm{do}^{20,21}$. Observa-se assim a necessidade de auxiliar na construção do conhecimento voltado aos $\mathrm{CP}$, para servir de suporte aos profissionais de saúde para que se sintam mais confiantes na prestação de cuidados a esses pacientes ${ }^{10}$.

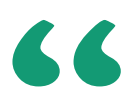

\section{O profissional enfermeiro contribui significativamente com os $C P$, todavia ele não desenvolve os cuidados sozinho, para isso conta uma equipe multidisciplinar.}

rio o fornecimento do apoio psicológico, social e espiritual ${ }^{5}$.

O despreparo dos profissionais, paciente e familiares, dificultam o entendimento e aceitação, portanto há necessidade de relações terapêuticas entre estes, com o intuito de construir parcerias, a fim de garantir a qualidade da assistência prestada. Para isso se faz necessário a comunicação clara em toda a trajetória da doença, enfatizando os planos de cuidado prévios, metas, a partir de uma avaliação cuidadosa, compassiva, visando o perfil de cada paciente são premissas para cuidado de excelência ${ }^{22,23}$.

Nesse escopo, é válida uma referência sobre a educação em saúde a familiares, como uma prática social, cujo processo "contribui para a sensibilização e formação da consciência crítica das pessoas a respeito de seus problemas de saúde, para, diante da sua realidade, discutir coletivamente, na busca de soluções" (p.89), junto à profissionais capacitados e a gestores. Envolvem ações sobre a formação de atitudes emancipadoras para a promoção da saúde, que se dá por meio da escuta ativa e do diálogo aberto, construindo relações horizontais, facilitadoras, que motivem as pessoas a colaborar na resolução de suas necessidades e exijam de seus governantes, ações potencializadoras de saúde ${ }^{24,25}$, atentas à promoção da saúde e à prevenção de agravos que expõem grupos vulneráveis.

\section{CONSIDERAÇÕES FINAIS}

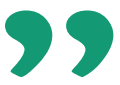

Entender os CP e reconhecer uma doença sem cura é extremamente difícil para o paciente e para as pessoas ao seu redor, sobretudo ao considerarmos que a reflexão sobre a morte ainda é vista como um sentimento de derrota, sendo pouco discutida e entendida em nossa sociedade. A morte é comumente considerada sinônimo de medo e tristeza diante de prognóstico desfavorável, sendo necessá-
Neste sentido, cabe a esses profissionais compreenderem os CP em sua ampla dimensão, ponderando a importância na APS para realizar ações assistenciais, burocráticas e educativas, criando estratégias para a implantação efetiva dos $\mathrm{CP}$, visando sempre o atendimento de qualidade ao paciente junto da equipe multidisciplinar e capacitação da equipe neste processo.

A partir desta reflexão, para que os profissionais possam compreender $\mathrm{CP}$ e consequentemente aplicar em sua práti- 
ca, acredita-se que devam haver ações voltadas à construção do conhecimento desses profissionais sobre o tema, visto que configurou-se como um dos grandes desafios encontrados neste estudo.

O processo de ensino e aprendizagem na graduação deve oferecer instrumentos potencializadores, metodologias ativas e transversalização dos saberes para fortalecer o conhecimento sobre os CP. Aos profissionais já graduados, ações de educação permanente sobre a temática deve fazer parte do planejamento do sistema de saúde, em todas as suas instâncias, incentivando ações intersetoriais e em rede, para que se favoreça não só o acesso, mas a resolutividade das necessidades de saúde dos usuários que precisam desses cuidados.

Embora os achados desta pesquisa sejam promissores, destaca-se algumas limitações, tais como o caráter regional da pesquisa. Nesse contexto, ressalta-se a importância que outros estudos sejam explorados nessa temática, como os CP na visão de usuários do sistema de saúde, oferecen- do novas contribuições para o desenvolvimento desta área do conhecimento.

Acredita-se que o estudo possa auxiliar os gestores municipais de saúde a perceberem os $\mathrm{CP}$ como estratégias na viabilização, condução e implementação de propostas inovadoras de cuidados alicerçadas nos preceitos protocolares do tema. A qualificação da atenção aos usuários dos serviços de saúde requer a implantação e efetividade das ações implementadas, devendo envolver trabalhadores, gestores, usuários, familiares.

\section{Referências}

1. Melo ACP. A polêmica da legalização da eutanásia no brasil: o dever ético de respeito às vontades antecipadas dos pacientes terminais. Jus Navegandi 2015 set [acesso em 25 mar 2018]. Disponível em: https://jus.com.br/artigos/42873. 2. World Health Organization. Better palliative care for older people. Geneva: World Health Organization; 2002.

3. Silveira MH, Ciampone MHT, Gutierrez BAO. Percepção da equipe multiprofissional sobre cuidados paliativos. Revista Brasileira de Geriatria e Gerontologia 2014 mar;17(1):7-16.

4. Brasil. Ministério da Sáude. Resolução nº 41, de 31 de outubro de 2018. Dispõe sobre as diretrizes para a organização dos cuidados paliativos, à luz dos cuidados continuados integrados, no âmbito Sistema Único de Saúde (SUS). 225. ed. Brasilia; 23 nov. 2018.

5. Santiago, FAO et al. Cuidados Paliativos na Atenção Primária: Conhecimento dos Médicos e Enfermeiros da Estratégia Saúde da Família, Revista Pesquisa em Saúde, abr 2019; 20(1): 16-19. Disponivel em: file:///C:/Users/cris_/Downloads/13164-43013-2-PB.pdf.

6. Neto ACM, Vale JMM do, Santos LMS dos, Santana ME de. 0 enfrentamento dos familiares cuidadores de adoecidos em cuidados paliativos oncológicos domiciliares diante dos estressores do cuidado. REAS [Internet]. 21 fev.2020 [citado 20jun.2020];12(2):e2525. Disponível em: https://acervomais.com.br/ index.php/saude/article/view/2525 .

7. Brasil. Conselho Nacional de Secretários de Saúde atualização junho/2015. Atenção Primária a Saúde. Para entender a gestão do SUS. Conselho Nacional de Secretários de Saúde. Brasília: Conass; 2015

8. Brasil. Ministério da Saúde (Org.). Política Nacional de Atenção Básica. PORTARIA N² 2.436, DE 21 DE SETEMBRO DE 2017[acesso em 01 nov 2017]. Disponível em: http://dab.saude.gov.br/portaldab/smp_como_funciona.php?conteudo=esf.

9. Santos DAS, Silva MS, Silva FP. A gestão de recursos naturais pelo enfermeiro na atenção básica. Centro de Ciências Naturais e Exatas - Ufsm 2015 jul;19(2):402-41.

10. Evangelista CB, Lopes MEL, Costa SFG, Abrão FMS, Batista PSS, Oliveira RC. Spirituality in patient care under palliative care: $A$ study with nurses. Escola Esc. Anna Nery 2016 [acesso em 15 abr 2018];20(1):176-182. Disponível em: http://dx.doi.org/10.5935/1414-8145.20160023.

11. Gomes ALZ, Othero MB. Cuidados paliativos. Estudos Avançados 2016 dez [acesso em 25 mar 2018];30(88):155-166. Disponível em: http://dx.doi. org/10.1590/s0103-40142016.30880011

12. Etkind SN, Bone AE, Gomes B, Lovell N, Evans CJ, Higginson, IJ et al. How many people will need palliative care in 2040 ? Past trends, future projections and implications for services. Bmc Medicine 2017 maio [acesso em 16 mar 2018];15(1):01-09. Disponível em: http://dx.doi.org/10.1186/s12916-0170860-2.

13. Marconi, MA, Lakatos, EM. Fundamentos de Metodologia Científica, $8^{\mathrm{a}}$ edição. 2017.Atlas.

14.Bardin, L. Análise de Conteúdo. Lisboa, 2009.Portugal; Edições 70, LDA.2009 15. Pereira, DG, Fernandes J, Ferreira LS, Rabelo RO, Pessalacia JDR, Souza RS. Significados dos cuidados paliativos na ótica de enfermeiros e gestores da atenção primária à saúde. Revista de Enfermagem UFPE On Line 2017 mar [acesso em 17 ago 2018]; 11(Supl. 3):1357-64. Disponível em: https://periodicos.ufpe.br/revistas/revistaenfermagem/article/viewFile/13977/16825.

16. Zimmermann, Camilla et al. Early palliative care for patients with advanced cancer: a cluster-randomised controlled trial. The Lancet, Elsevier; maio 2014 383(9930);1721-1730,. Elsevier BV. Disponível em: http://dx.doi.org/10.1016/ s0140-6736(13)62416-2.

17. Meneguin S, Ribeiro R. Dificuldades de cuidadores de pacientes em cuidados paliativos na estratégia da saúde da família. Texto \& Contexto - Enfermagem 2016 [acesso em 22 mar 2018];25(1):02-07. Disponível em: http://dx.doi. org/10.1590/0104-0707201500003360014 .

18. Combinato, Denise Stefanoni; Ferreira Martins, Sueli Terezinha. (Em defesa dos) Cuidados Paliativos na Atenção Primária à Saúde. Mundo da Saúde, 2012; 36(3): 433-441, Disponível em: http://hdl.handle.net/11449/73427.

19. Capelas ML, Silva SCFS, Alvarenga MISF, Coelho SP. Cuidados paliativos: 0 que é importante saber. Patient Care 2016 maio [acesso em 29 mar 2018]:1620. Disponível em:https://www.researchgate.net/profile/Manuel_Capelas/ publication/305659147 Cuidados Paliativos 0 que e importante_saber/ links/57989fc108aebOffcd089a34/Cuid ados-Paliativos-0-que-e-importante-saber.pd.

20. Zaman S, Inbadas H, Whitelaw A, Clark D. Common or multiple futures for end of life care around the world? Ideas from the 'waiting room of history'. Social Science \& Medicine 2017 jan [acesso em 22 mar 2018];172:72-79. Disponível em: http://dx.doi.org/10.1016/j.socscimed.2016.11.012.

21. Sawatzky, R. et al. Conceptual foundations of a palliative approach: a knowledge synthesis. Bmc Palliative Care, Springer Nature jan. 2016; 15(1)1-6. Disponível em: http://dx.doi.org/10.1186/s12904-016-0076-9.

22. Cobo, VA, Fabroo, AL, Parreira, AC, Pardi,F. Cuidados Paliativos na atenção Primária à Saúde: prespectiva dos profissioanis de saúde.Boletim Academia Paulista de Psicologia. 2019. V.39, nº97,p.225-235.São Paulo, Brasil.

23. Santiago, FAO, Brito, LMO, Martins, DMF, Barbosa, MCL. et.al. Cuidados paliativos na Atenção Primária: conhecimento dos médicos e enfermeiros da estratégia Saúde da Família. Revista Pesquisa em Saúde, 2019. 20(1):1619,2019. São Luis, Maranhão, Brasil.

24. Atty, ATM, Tomazelli, JG. Cuidados Paliativos na atenção domiciliar para pacientes oncológicos no Brasil. Revista Saúde em Debate, 2018, V.42,nº116, p.225-236. Rio de Janeiro, Brasil.

25. Fontana R. 0 processo de educação em saúde para além do hegemônico na prática docente. Revista Contexto e Educação. 2018; 33(106); 84-98. Disponível em: https://doi.org/10.21527/2179-1309.2018.106.84-98 . 